\title{
Serine catabolism is essential to maintain mitochondrial respiration in mammalian cells
}

\author{
Stephanie Lucas ${ }^{1}$, Guohua Chen ${ }^{1}$, Siddhesh Aras $^{2}$, Jian Wang ${ }^{1,3}$ (1)
}

\begin{abstract}
Breakdown of serine by the enzyme serine hydroxymethyltransferase (SHMT) produces glycine and one-carbon (1C) units. These serine catabolites provide important metabolic intermediates for the synthesis of nucleotides, as well as methyl groups for biosynthetic and regulatory methylation reactions. Recently, it has been shown that serine catabolism is required for efficient cellular respiration. Using CRISPR-Cas9 gene editing, we demonstrate that the mitochondrial SHMT enzyme, SHMT2, is essential to maintain cellular respiration, the main process through which mammalian cells acquire energy. We show that SHMT2 is required for the assembly of Complex I of the respiratory chain. Furthermore, supplementation of formate, a bona fide $1 \mathrm{C}$ donor, restores Complex I assembly in the absence of SHMT2. Thus, provision of $1 \mathrm{C}$ units by mitochondrial serine catabolism is critical for cellular respiration, at least in part by influencing the assembly of the respiratory apparatus.
\end{abstract}

DOI 10.26508/Isa.201800036 | Received 22 February 2018 | Revised 7 May 2018 | Accepted 8 May 2018 | Published online 21 May 2018

\section{Introduction}

Apart from its role in protein synthesis, serine is a major metabolic source for generating one-carbon (1C) units in mammalian cells (de Koning et al, 2003). Two serine hydroxymethyltransferase (SHMT) enzymes, SHMT1 and 2, break down serine into glycine and methylene-tetrahydrofolate (THF) in the cytosol and mitochondria, respectively (Stover \& Schirch, 1990; Stover et al, 1997). The latter serine catabolite feeds into cellular $1 \mathrm{C}$ pool, and either directly participates in thymidine synthesis or indirectly in purine or methionine synthesis after its oxidative or reductive conversion to formyl- or methyl-THF (Tibbetts \& Appling, 2010). Because the 1Cderived products are key anabolic building blocks, sustaining the $1 C$ pool is vital for cellular proliferation and is required for a number of physiological and pathophysiological processes ranging from stem cell renewal to cancer progression (Wang et al, 2009; Locasale, 2013). Consistent with their critical roles in supporting cell proliferation, SHMTs are highly active in many rapidly growing cancer cells and are important molecular targets for cancer intervention (Snell et al, 1988; Nikiforov et al, 2002; Ducker et al, 2017).

Interestingly, 1C metabolism also functionally interacts with mitochondrial oxidative phosphorylation (OXPHOS) system, the main process through which mammalian cells generate ATP. The OXPHOS system comprises an electron transport chain of four respiratory enzyme complexes (Complex I-IV) that use nutrientderived redox potentials to drive Complex V (CV), the ATP synthase (Alberts et al, 2002). The protein components of the OXPHOS system are encoded by both nuclear and mitochondrial genes (Ott et al, 2016). It was recently shown that electron transport chain dysfunction owing to mitochondrial DNA (mtDNA) depletion dramatically alters the expression of SHMT2 as well as the production of $1 \mathrm{C}$ units from serine catabolism (Bao et al, 2016; Nikkanen et al, 2016). In addition, system-wide metabolic modeling indicates that oxidation of the serine-derived $1 \mathrm{C}$ units provides a significant fraction of the redox potential to drive ATP synthesis via OXPHOS (Vazquez et al, 2011; Tedeschi et al, 2013). These observations strongly suggest that the $1 \mathrm{C}$ metabolic cycle and the OXPHOS system are functionally coupled. Recent studies further demonstrated that serine catabolism by SHMT2 is required to maintain mitochondrial respiration in human cell lines (Minton et al, 2018; Morscher et al, 2018) and mouse tissues (Tani et al, 2018). Interestingly, these works revealed distinct mechanisms underlying a crucial role of SHMT2 in sustaining mitochondrial translation in different cell types (Minton et al, 2018; Morscher et al, 2018), indicating that complex mechanisms exist, linking serine catabolism to the modulation of the OXPHOS system.

In the present study, we independently investigated the metabolic adaptions in response to targeted deletion of SHMT enzymes in mammalian cells. Consistent with the previous reports (Minton et al, 2018; Morscher et al, 2018), we found that the cells lacking SHMT2, but not SHMT1, preferentially metabolized glucose to lactate and were unable to survive in the presence of galactose media, suggestive of mitochondrial dysfunction in the absence of SHMT2. Mechanistically, we found that SHMT2 is dispensable for mtDNA maintenance and OXPHOS gene expression. However, our results strongly suggest that SHMT2 plays a critical role in supporting the assembly of Complex I by supplying the $1 \mathrm{C}$ intermediate derived

\footnotetext{
Department of Pathology, Wayne State University, Detroit, MI, USA ${ }^{2}$ Center for Molecular Medicine and Genetics, Wayne State University, Detroit, MI, USA ${ }^{3}$ Cardiovascular Research Institute, Wayne State University, Detroit, MI, USA
}

Correspondence: jianwang@med.wayne.edu 
from serine catabolism. Together, our findings revealed a novel regulatory link between SHMT2-mediated $1 \mathrm{C}$ metabolism and the maintenance of the mitochondrial respiratory chain in mammalian cells.

\section{Results}

\section{Loss of SHMT2 stimulates aerobic glycolysis}

To examine the function of SHMT1 and SHMT2 enzymes, each gene was independently ablated in 293A cells using CRISPR-Cas9 technology. Two mutant cell lines for each SHMT gene were generated using two different small-guide RNAs (sgRNAs) to target each SHMT gene at different genomic locations. Transfections of Cas9 alone into the human embryonic kidney 293A (HEK293) cells did not affect SHMT expression and were used as the WT control. Both SHMT1

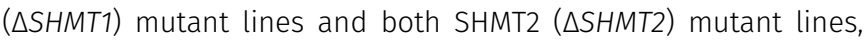
created by introduction of the gRNAs, showed complete loss of the respective protein, as determined by the Western blotting analysis (Fig 1A).

Consistent with a previous report (Ducker et al, 2016), both $\triangle S H M T 1$ and $\triangle S H M T 2$ cells grew similarly to the WT when maintained in the standard DMEM media, which contains high levels of glucose (Fig 1B). This may reflect a redundancy of SHMT paralogs to supply $1 C$ units for supporting cell proliferation (Ducker et al, 2016). Interestingly, it was noted that $\triangle S H M T 2$ cells consistently turned the culture media acidic faster than the WT or $\triangle S H M T 1$ cells, suggesting an elevated rate of glycolysis as a result of SHMT2 loss. This possibility was supported by the observation that $\triangle S H M T 2$ cells had significantly increased production of lactic acid, the end product of aerobic glycolysis, whereas $\triangle S H M T 1$ cells did not (Fig 1C). In addition, the levels of key glycolytic enzymes, including pyruvate kinase $M(P K M)$ and lactate dehydrogenase A, were modestly elevated in the $\triangle S H M T 2$ cells (Fig 1A). Thus, these results indicate that the cells lacking SHMT2 prefer partial metabolism of glucose to lactate.

\section{SHMT2 is essential for maintaining mitochondrial respiration}

Up-regulation of aerobic glycolysis can indicate a metabolic adaption to mitochondrial dysfunction (Wu et al, 2007). To test this possibility, we forced the cells to rely on mitochondrial respiration by growing the cells in media containing galactose instead of glucose (Robinson et al, 1992). Under these conditions, we observed that the $\triangle S H M T 2$, but not the $\triangle S H M T 1$, cells exhibited reduced intracellular ATP and were unable to proliferate (Fig 2A and B), F2 indicating mitochondrial dysfunction in the absence of SHMT2. In contrast, the $\triangle S H M T 2$ cells were able to maintain a normal intracellular ATP level in media containing glucose (Fig 2C). However, we observed a significant reduction of basal oxygen consumption by the $\triangle S H M T 2$ cells when maintained in standard glucose media (Fig 2D). Together, these results demonstrate that the mitochondrial serine catabolic enzyme SHMT2 is essential for maintaining normal levels of cellular respiration.
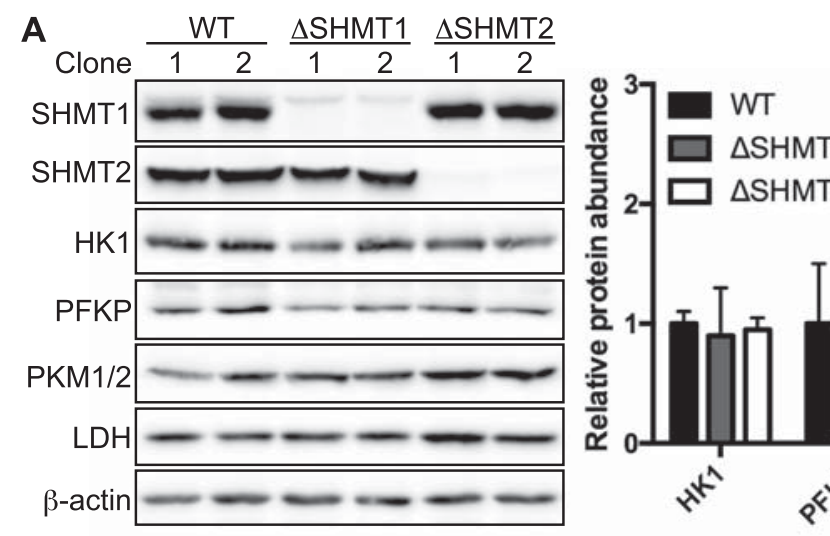

\section{1}

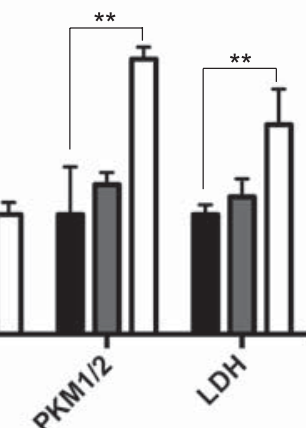

B

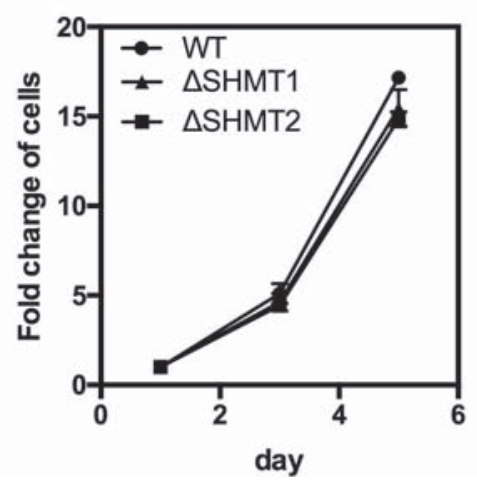

C

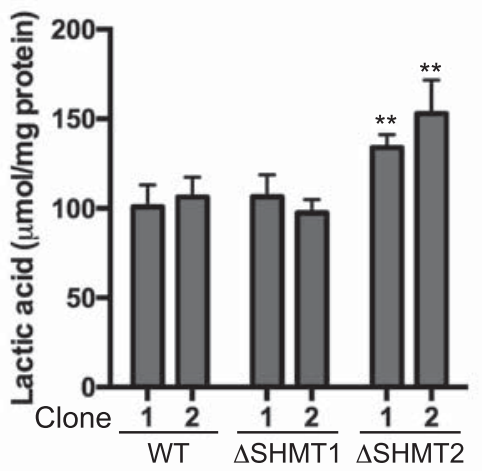

Figure 1. Effects on glycolysis by the deletion of serine catabolic enzymes in 293A cells.

(A) Protein levels of SHMT1, SHMT2, hexokinase 1 (HK1), phosphofrutokinase (platelet type, PFKP), pyruvate kinase (muscle type $1 / 2, \operatorname{PFKM} 1 / 2$ ), lactate dehydrogenase $A(L D H A)$, and $\beta$-actin were measured in the WT, $\triangle$ SHMT1, or $\triangle$ SHMT2 293A cells by Western blotting. Two independent cell clones of each genotype were examined. Densitometry quantification of the glycolytic proteins followed by normalization to $\beta$-actin is plotted on the right. ${ }^{* *} P<$ 0.01 ( $t$ test). Data are presented as mean \pm SD for three independent experiments. (B) Proliferation rates of the WT, $\triangle$ SHMT1, and $\triangle S H M T 2293 \mathrm{~A}$ cells in the DMEM with $4.5 \mathrm{~g} /$ liter glucose. Data are presented as mean \pm SD $(n=5)$. (c) Measurement of the lactic acid production from the WT, $\triangle$ SHMT1, and $\triangle$ SHMT2 293A cells that were grown in DMEM for $48 \mathrm{~h}$. Data are presented as mean $\pm S D(n=4){ }^{* *} P<0.01$ ( $t$ test). 
A
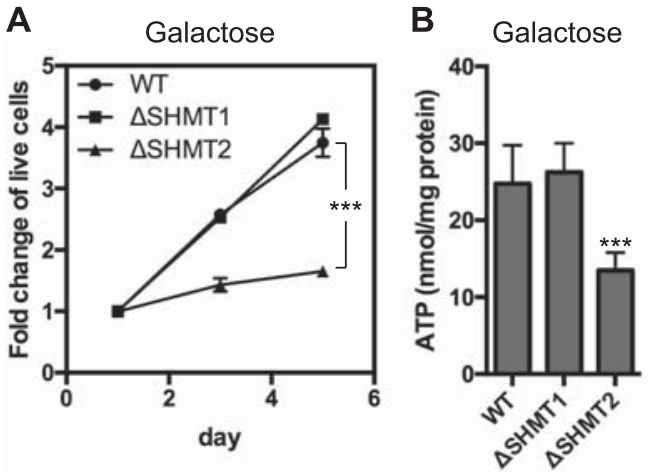

E

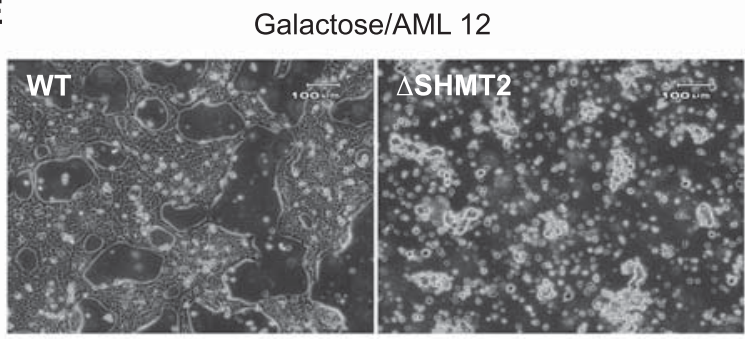

C

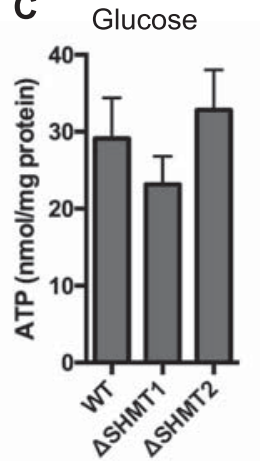

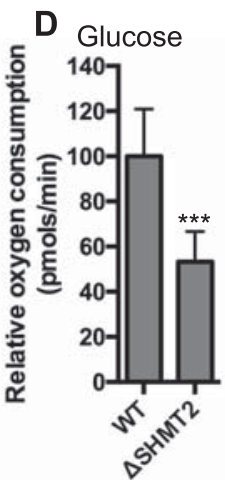

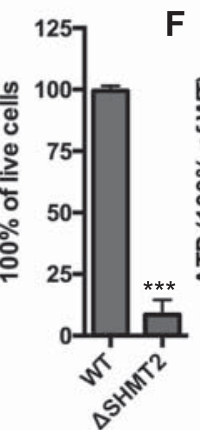

$\mathbf{F}$

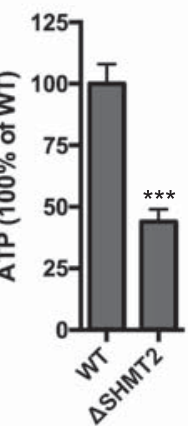

Figure 2. Effects on mitochondrial respiration by the deletion of serine catabolic enzymes in 293A and AML12 cells.

(A) Measurement of the cell proliferation of the WT, $\triangle S H M T 1$, and $\triangle S H M T 2293 \mathrm{~A}$ cells in the DMEM-based galactose media. (B, C) Measurement of the intracellular ATP levels in the WT, $\triangle S H M T 1$, and $\triangle S H M T 2$ 293A cells that were grown in the galactose (B) or glucose (C) media for $24 \mathrm{~h}$. (D) Measurement of the basal oxygen consumption rates of the WT and $\triangle$ SHMT2 293A cells that were grown in the glucose media. (E) Phase-contrast images illustrating the WT and $\triangle$ SHMT2 AML12 cells that were grown in the galactose media for $72 \mathrm{~h}$. The percentages of the live cells were plotted on the right. (F) Measurement of the intracellular ATP levels of the WT and $\triangle$ SHMT2 AML12 cells that were grown in the galactose media for $72 \mathrm{~h}$. ${ }^{* * *} P<0.001$ ( $t$ test). Data are presented as mean \pm SD. $n$ $=5$ for $(A) ; n=4$ for $(B-E)$; and $n=6$ for $(F)$.

To test whether the dependence of cellular respiration on SHMT2 extends to another cell type, we deleted SHMT2 in the AML12 mouse hepatocyte using CRISPR-Cas9 genome editing system. Consistent with the observations in 293A cells, knockout of SHMT2 in AML12 cells led to cell death (Fig 2E) and a decrease in intracellular ATP levels (Fig 2F) in the presence of galactose media. Thus, the essential role for SHMT2 to maintain respiration is conserved between two different cell types from different species (human and mouse).

\section{SHMT2 is dispensable for the maintenance of mtDNA content and OXPHOS gene expression}

To test whether loss of SHMT2 affects the expression of the individual OXPHOS enzyme complexes, we subjected the WT and mutant 293A cells to Western blotting analysis with Total OXPHOS antibody cocktail. Interestingly, we observed that knockout of SHMT2 selectively decreased the level of Complex I as revealed by its marker NADH:ubiquinone oxidoreductase subunit B8 (Fig 3A). Moreover, such decrease in Complex I level in the $\triangle S H M T 2$ cells was further confirmed by measuring NADH:ubiquinone oxidoreductase core subunit S1 (NDUFS1), a core subunit of Complex I (Fig 3A). These results indicate that cells lacking SHMT2 have a defect in the mitochondrial OXPHOS system, which is particularly associated with the Complex I.

The mammalian mitochondrial genome encodes 13 peptides that are core components of the OXPHOS system (Neupert, 2016). Loss of SHMT2 reduces cellular deoxynucleotide pool (Ducker et al, 2016), which can be associated with mtDNA depletion (Bourdon et al, 2007; Suomalainen \& Battersby, 2018). However, when we measured mtDNA copy numbers, we observed that the $\triangle S H M T 2$ cells had similar mtDNA content to the WT and $\triangle S H M T 1$ cells (Fig 3B). Next, we determined the relative mRNA expression of the OXPHOS system genes in the $\triangle S H M T 2$ cells to the WT, by next-generation sequencing (for the nuclear-encoded transcripts) and by quantitative RT-PCR (qRT-PCR) analysis (for the mitochondria-encoded transcripts). The results showed that knockout of SHMT2 did not reduce the expression of any mRNA species that encodes the OXPHOS enzyme (Fig 3C and D). The cellular $1 \mathrm{C}$ pool connects to the synthesis of formyl-methionyl-tRNA, a metabolite that is required for the initiation of mitochondrial translation (Kozak, 1983). This raises the possibility that loss of SHMT2 might reduce the synthesis of the mitochondriaencoded proteins. However, when we measured mitochondrial protein synthesis by metabolically labeling the cells with ${ }^{35} \mathrm{~S}$-methione/ cysteine in the presence of the cytosolic protein translation inhibitor emetine, we observed that the ability of the $\triangle S H M T 2$ cells to synthesize the mitochondria-encoded proteins was comparable with the WT (Fig 3E). This is in contrast to the recent observations that deletion of SHMT2 negatively impacted mitochondrial translation in the Jurket and HCT116 cells (Minton et al, 2018; Morscher et al, 2018). Although a possible negative impact by SHMT2 deletion on mitochondrial translation cannot be entirely excluded in our cell model, our results suggest that the regulation of the OXPHOS system by SHMT2 may also occur after the synthesis of mitochondria-encoded OXPHOS proteins.

\section{Loss of SHMT2 severely reduces the level of mature Complex I}

We next tested whether deletion of SHMT2 influences the expression of the individual mature OXPHOS complexes. Blue native gel electrophoresis (BNGE) allows for the separation of multiprotein complexes in a native conformation with high resolution (Schägger \& von Jagow, 1991). Using BNGE to examine the steady-state levels of the mature OXPHOS complexes in the WT and $\triangle$ SHMT2 293A cells, we observed that loss of SHMT2 essentially abolished the expression of the monomeric Complex I and the Complex III dimer $\left(\mathrm{CII}_{2}\right) / \mathrm{CIV}$ supercomplex (Fig 4A). In contrast, the levels of the $\mathrm{CII}_{2}$ and $\mathrm{CV}$ F4 


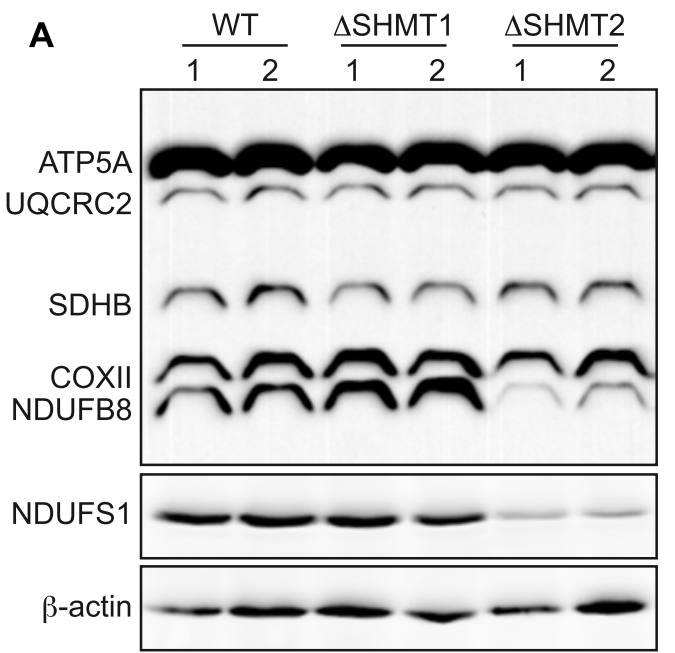

D

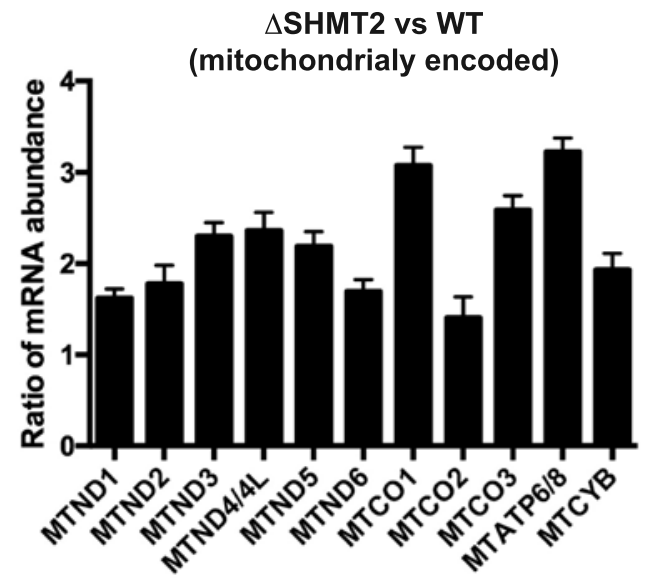

B

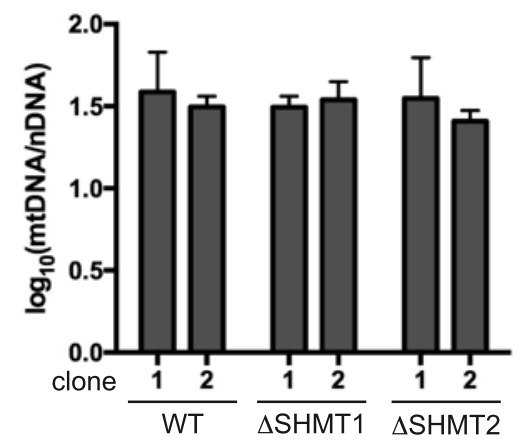

C (nuclearly encoded)

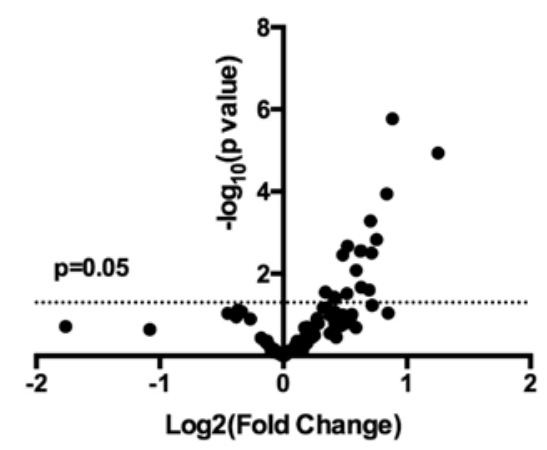

E

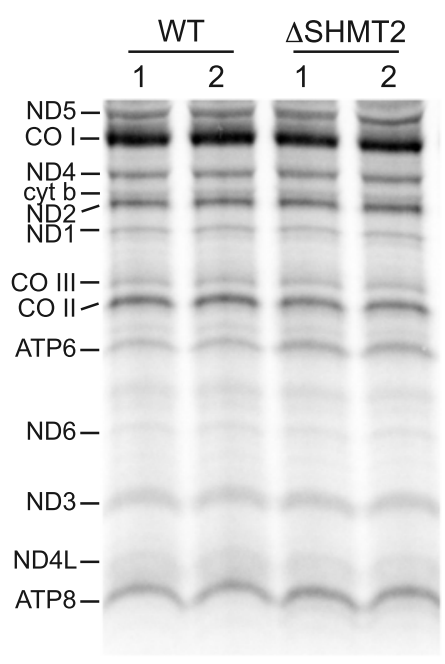

${ }^{35} \mathrm{~S}$ pulse

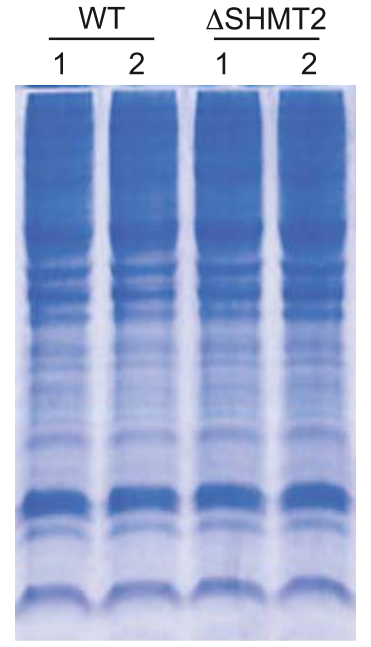

Coomassie staining

Figure 3. Effects on the content of mtDNA and the expression of the OXPHOS genes by deletion of SHMT2 in $293 \mathrm{~A}$ cells.

(A) Measurement of the levels of the respiratory chain complexes (RCCS) in the WT, $\triangle S H M T 1$, and $\triangle S H M T 2293$ A cells by Western blotting, using the Total OXPHOS antibody cocktail to simultaneously detect the representative components of each individual RCCs: Cl-NADH:ubiquinone oxidoreductase subunit B8; ClI-succinate dehydrogenase complex subunit B; CIII-ubiquinol-cytochrome C reductase core protein 2; CIV-cytochrome C oxidase II; and CV-ATP synthase F1 subunit alpha. NDUFS1, an additional CI marker, and $\beta$-actin, the loading control, were also measured. (B) Bar graph illustrating the mtDNA copy numbers of the WT, $\triangle S H M T 1$, and $\triangle S H M T 2293 \mathrm{~A}$ cells. Data are presented as mean \pm SD ( $\mathrm{n}=4$ ). (C) Scatter plot illustrating the log2-transformed relative mRNA levels of the nuclear-encoded OXPHOS genes in the $\triangle S H M T 2293 \mathrm{~A}$ cells to the WT control, as determined by next-generation sequencing; $t$ test $(n=3)$. (D) Bar graph illustrating the relative mRNA levels of the mitochondria-encoded OXPHOS genes in the $\triangle$ SHMT2 $293 \mathrm{~A}$ cells to the WT control, as determined by qRT-PCR analysis. Data are presented as mean \pm SD $(n=3)$. (E) Metabolic radiolabeling to determine the synthetic rates of the mitochondria-encoded proteins in the WT and $\triangle$ SHMT2 293A cells. Left, autoradiography to visualize the mitochondria-encoded OXPHOS proteins; right, Coomassie blue staining of total cellular proteins.

monomer were unaffected in the $\triangle S H M T 2$ cells (Fig 4A). The dramatically decreased Complex I level and activity were further revealed in the $\triangle S H M T 2$ cells using a Western blotting analysis probed with NDUFS3 antibody (Fig 4B) and an in-gel NADH oxidation assay (Fig 4C), respectively. In conjunction with the analyses on the levels of the monomeric CII and CIV (data shown below), we conclude that loss of SHMT2 selectively impedes the assembly of mature Complex I and the $\mathrm{CIII}_{2}$ / CIV supercomplex in 293A cells.

\section{Reconstitution of SHMT2 restores Complex I in the SHMT2- deficient cells}

To firmly establish the causative relationship between SHMT2 expression and Complex I functionality, we re-expressed SHMT2 into the $\triangle$ SHMT2 293A cells. The results showed that exogenous expression of SHMT2 protein in the $\triangle$ SHMT2 cells to $~ 70 \%$ of the WT level led to significant recovery of Complex I level (Fig S1A) and activity (Fig S1B), as well as improvement of cell survival in galactose media (Fig S1C). Thus, genetic restoration strongly supports the conclusion that SHMT2 is required for Complex I assembly.

\section{Formate supplementation restores Complex $I$ in the SHMT2-deficient cells}

Loss of SHMT2 leads to glycine auxotrophy (Pfendner \& Pizer, 1980) and depletion of formyl-THF (Ducker et al, 2016). To test whether serine catabolites are involved in the regulation of Complex I, we supplemented the $\triangle$ SHMT2 293A cells with the increasing amounts 


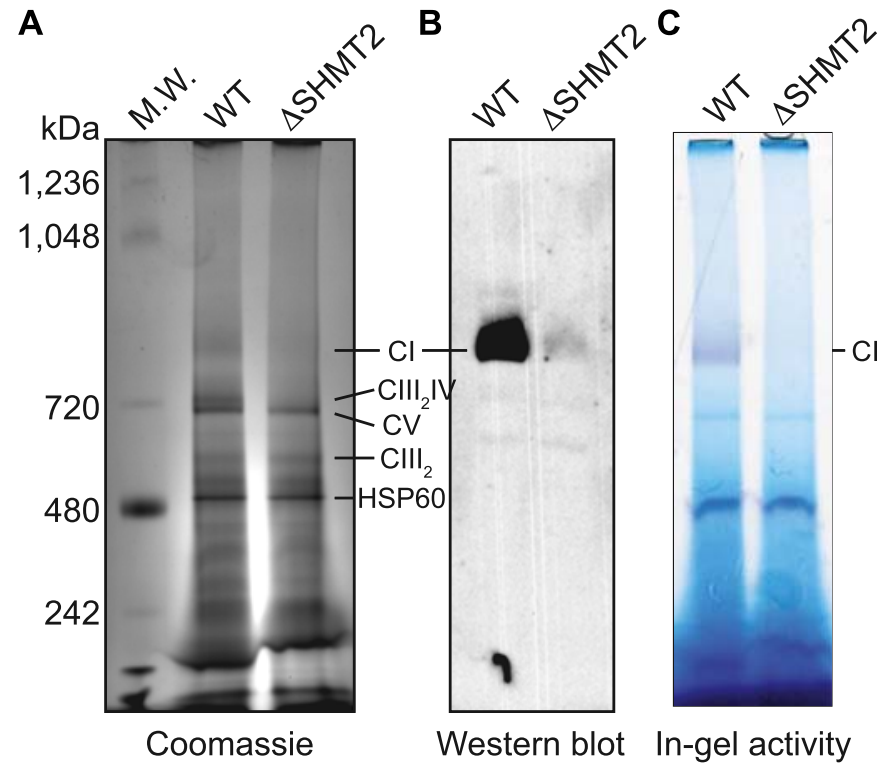

Figure 4. Effects on the assembly of mature respiratory chain complexes by the deletion of SHMT2.

(A-C) BNGE to detect individual mature respiratory complexes in the mitochondrial preparation from the WT and $\triangle$ SHMT2 293A cells, followed by visualization with Coomassie blue staining (A), Western blotting with the antibody against a Complex I (Cl) marker NDUFS3 (B), or Complex I in-gel activity assay (C). Cl, Complex I; $\mathrm{CIII}_{2} / \mathrm{IV}, \mathrm{CIII}_{2} /$ IV supercomplex; HSP60, heat shock protein 60.

of glycine or formate, a bona fide $1 \mathrm{C}$ donor (Brosnan \& Brosnan, 2016). We observed that formate, but not glycine, significantly increased the levels of NDUFS1, a core subunit of Complex I, in a timeand dose-dependent manner (Fig 5A and B). We next determined how formate treatment influences the expression of the individual mature OXPHOS complexes. Using BNGE analyses, we demonstrated that loss of SHMT2 selectively abolished the expression of mature Complex I without affecting that of the other four individual complexes, including $\mathrm{CII}, \mathrm{CIII}_{2}$, CIV, and CV (Fig 5C, lane 1 versus 3). Importantly, formate treatment completely restored Complex I assembly (Fig 5C) and activity (Fig 5D) in $\triangle S H M T 2$ cells, as well as the proliferation of $\triangle S H M T 2$ cells in galactose (Fig 5E). These results strongly suggest that the effect of SHMT2 loss on Complex I assembly is mediated by altered mitochondrial $1 \mathrm{C}$ metabolism.

\section{Discussion}

Serine is uniquely positioned at a metabolic crossroad that connects the major catabolic and anabolic pathways (de Koning et al, 2003; Kalhan \& Hanson, 2012). Although synthesized from a glycolytic catabolite, it can be broken down to supply key anabolic intermediates for the synthesis of a number of important biomolecules, including nucleotides, lipids, and glutathione. It is not surprising, therefore, that serine itself is a key regulator of central metabolic pathways. This role is well illustrated by the allosteric regulation of serine on PKM2-a key glycolytic enzyme whose role is to partition glucose carbon into biosynthetic pathways in cancer cells (Chaneton et al, 2012; Ye et al, 2012). Here, we find that catabolism of serine by
SHMT2 is a regulatory determinant of cellular respiration. Moreover, we further demonstrate that the $1 \mathrm{C}$ units produced by SHMT2-mediated serine catabolism are essential for the assembly of functional Complex I. Our investigations, thus, uncovered a novel metabolic checkpoint for the assembly of the respiratory apparatus, suggesting that transformation of serine into $1 \mathrm{C}$ units coordinates the bioenergetic and biosynthetic pathways in mammalian cells.

Cellular adaptions to the loss of the serine-catabolic pathway either in the cytosol or mitochondria were investigated in the present study. We observed that loss of the SHMT enzyme in either cellular compartment posed minor impact on cell proliferation when the cells were grown in complete media. This is consistent with previous reports that SHMT paralogs are redundant for supply of $1 C$ units in support of cell proliferation (Bao et al, 2016; Ducker et al, 2016). Interestingly, we observed that loss of SHMT2, but not SHMT1, led to elevation of aerobic glycolysis, indicating the involvement of SHMT2 in retrograde regulation on glycolysis in the cytosol. However, the up-regulation of glycolysis in response to SHMT2 deficiency cannot be easily explained by the allosteric activation of PKM2 by serine (Chaneton et al, 2012; Ye et al, 2012) because cellular serine levels were shown to be unaltered in the HEK293 cells lacking SHMT2 in previous studies (Bao et al, 2016; Ducker et al, 2016). Instead, our findings suggest that such elevation of glycolysis is a compensatory response to mitochondrial dysfunction. This compensation allows the substrate-level phosphorylation of glycolysis to substitute energy production from mitochondrial respiration, as previously observed in some cancer cells (Wu et al, 2007). Our observation made in 293A cells is consistent with the previous observations that SHMT2, but not SHMT1, is essential for maintaining mitochondrial respiration in the Jurket and HCT116 cells (Minton et al, 2018; Morscher et al, 2018). The results of the present study support the conclusion that SHMT2 is a critical metabolic checkpoint for OXPHOS function in a variety of mammalian cell types.

As a major metabolic route to produce $1 \mathrm{C}$ units, serine catabolism by SHMT2 might influence the OXPHOS gene expression in several ways including the influence on (i) cellular nucleotide homeostasis (Bao et al, 2016; Ducker et al, 2016) and therefore mtDNA replication (Anderson et al, 2011); (ii) mRNA expression of the OXPHOS genes (Mentch et al, 2015; Kottakis et al, 2016); and (iii) biosynthesis of the mitochondria-encoded OXPHOS proteins (Minton et al, 2018; Morscher et al, 2018). Recent studies demonstrated that SHMT2 is necessary for sustaining mitochondrial translation in the Jurket human leukemic (Minton et al, 2018) and HCT116 human colon cancer (Morscher et al, 2018) cells. Interestingly, these studies revealed distinct mechanisms that account for different types of translational abnormalities consequent to SHMT2 deletion in these two cell types. In response to SHMT2 deletion in Jurket cells, Minton et al (2018) found a decrease in formyl-methionyl-tRNA synthesis. This resulted in impaired mitochondrial translation initiation, leading to defective global OXPHOS protein synthesis in mitochondria (Minton et al, 2018). Morscher et al (2018), on the other hand, found that SHMT2 deletion in HCT116 cells caused a reduction in the methylation of certain tRNA species. This resulted in ribosomal stalling in HCT116 cells, leading to defective translation for a subset of the mitochondria-encoded 
A
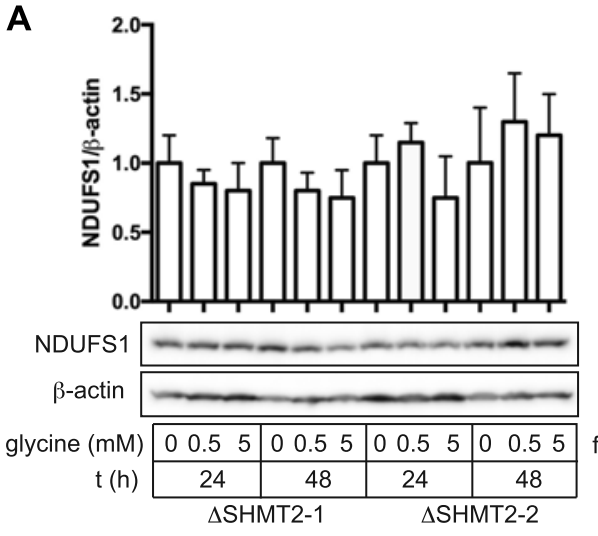

B
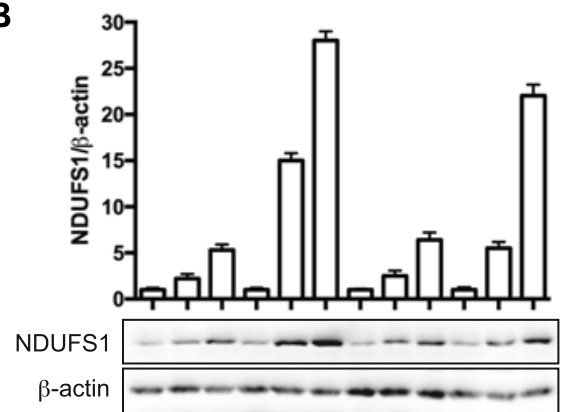

formate $(\mathrm{mM}) \quad$\begin{tabular}{lll|lll|lll|lll}
0 & 0.5 & 2 & 0 & 0.5 & 2 & 0 & 0.5 & 2 & 0 & 0.5 & 2 \\
\hline
\end{tabular}

$t(h)$

D

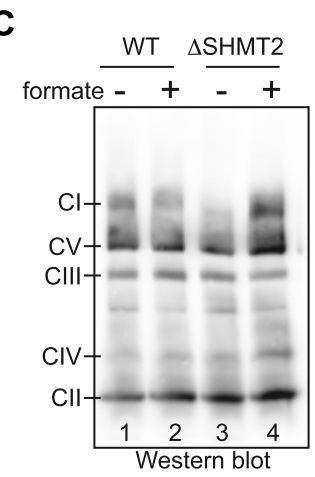

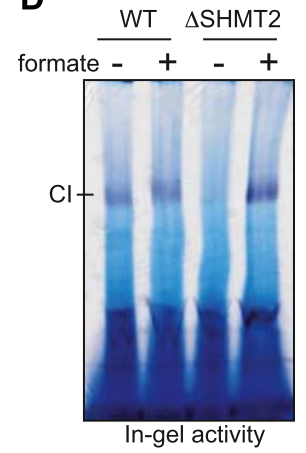

$\mathbf{E}$

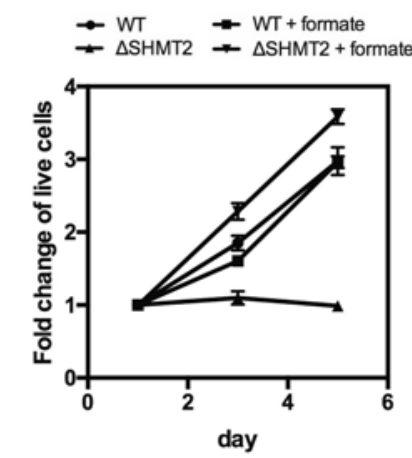

Figure 5. Effects of glycine and formate on the assembly of Complex I.

(A, B) Time- and dose-dependent effects on the levels of Complex I core subunit NDUFS1 by the supplementation of the $\triangle S H M T 2293$ A cells with glycine (A) or formate (B). Densitometry quantification of NDUFS1 followed by normalization to $\beta$-actin is plotted on the top. Data are presented as mean \pm SD for three independent experiments. (C, D) BNGE resolution of the mitochondrial preparation from the WT and $\triangle S H M T 2293$ A cells that were treated with or without $2 \mathrm{mM}$ formate for $72 \mathrm{~h}$, followed by visualization of the individual mature respiratory chain complexes by the Western blotting probed with the Total OXPHOS antibody cocktail (C) and the Complex I (CI) in-gel activity assay (D). Cl, Complex I. (E) Proliferation rates of the WT and $\triangle$ SHMT2 293A cells in the galactose media supplemented with or without $2 \mathrm{mM}$ formate. Data are presented as mean \pm SD $(n=4)$.
OXPHOS proteins (Morscher et al, 2018). Consistent with these previous reports, our results showed that deletion of SHMT2 did not decrease the mtDNA content or the expression of the mRNAs that encode OXPHOS enzymes. Surprisingly, using ${ }^{35}$ S-methionine/ cysteine pulse labeling, we were unable to observe an appreciable alteration in the biosynthesis of the mitochondria-encoded OXPHOS proteins in the SHMT2-deficient 293A cells. Given that differential translational responses to SHMT2 deletion were also observed from the different cell types in the previous studies (Minton et al, 2018; Morscher et al, 2018), it is plausible that SHMT2 could modulate mitochondrial translation in a cell-type-specific manner. Nevertheless, we cannot entirely exclude the possibility that loss of SHMT2 could pose a negative impact on the mitochondrial translation in 293A cells. A more sophisticated approach such as ribosome profiling (Ingolia, 2016; Morscher et al, 2018) is desired to further investigate this matter in 293A cells. Interestingly, we further found that deletion of SHMT2 selectively and dramatically reduced the steady-state levels of the mature Complex I and the $\mathrm{CIII}_{2} / \mathrm{CIV}$ supercomplex, and that exogenous $1 \mathrm{C}$ supplementation restored the assembly of Complex I in the SHMT2-deficient 293A cells. These results raise the novel possibility that the $1 C$ metabolic intermediate derived from mitochondrial serine catabolism is a metabolic determinant for the assembly of respiratory chain complex, in addition to its contribution to mitochondrial translation.

Assembly of the respiratory chain determines the competence of the OXPHOS system. However, the molecular mechanism for this process is far from understood (Stroud et al, 2016; Guerrero-Castillo et al, 2017). Although our findings demonstrate that SHMT2mediated $1 \mathrm{C}$ metabolism is required for Complex I assembly, the exact mechanism by which this occurs is unknown and requires further investigation. Interestingly, it has been recently shown that ribosome stalling mostly impaired Complex I activity in the absence of SHMT2 (Morscher et al, 2018). This raises the question of whether lack of mitochondrial serine catabolism influences potential functional interaction between mitochondrial translation and complex assembly. However, it could also be speculated that the metabolites derived from $1 \mathrm{C}$ pool may convey a regulatory signal to molecular factor(s) involved in complex assembly. As such, some Complex I assembly factors, for example, the NADH:ubiquinone oxidoreductase complex assembly factor 5 and the NADH:ubiquinone oxidoreductase subunit A9 were found to harbor S-adenosylmethionine and NADPH (Rhein et al, 2013; Fiedorczuk et al, 2016), two important downstream metabolites derived from the $1 C$ cycle (Tibbetts \& Appling, 2010; Ducker \& Rabinowitz, 2017). It is, thus, interesting to further test whether these factors might influence Complex I assembly in response to the fluctuations of cellular $1 \mathrm{C}$ pool. In addition, the results presented here provoke questions beyond the mechanistic aspects of how $1 \mathrm{C}$ metabolites are involved in Complex I assembly. For example, does the fluctuation of the $1 \mathrm{C}$ pool dynamically regulate Complex I assembly in a temporal-spatial manner? Is this regulation also important for the maintenance of metabolic homeostasis at a systemic level? As such, the findings made here may also suggest that targeting serine catabolism could be useful for intervening in a broad spectrum of the human pathologies associated with mitochondrial dysfunction, in addition to cancer. 


\section{Materials and Methods}

\section{Cell culture, plasmid construction, and mutant cell line establishment}

HEK293A (Invitrogen) and 293T (American Type Culture Collection) cells were maintained in DMEM medium supplemented with $10 \%$ FBS, $100 \mathrm{U}$ of penicillin $/ \mathrm{ml}$, and $0.1 \mathrm{ng}$ of streptomycin $/ \mathrm{ml}$. Murine AML12 hepatocytes (American Type Culture Collection) were maintained in a 1:1 mixture of DMEM and Ham's F12 medium, supplemented with 10\% FBS, 1:100 insulin-transferrin-selenium (Invitrogen), $100 \mathrm{U}$ of penicillin/ml, and $0.1 \mathrm{ng}$ of streptomycin $/ \mathrm{ml}$.

For construction of the targeting vector against human SHMT, the sgRNA sequences were determined using a web bioinformatics tool (http:// crispr.mit.edu) (Ran et al, 2013). The oligo DNAs that encode sgRNA were annealed and cloned into pSpCas9(BB)-2A-Bsd, a modified vector from pSpCas9(BB)-2A-Puro (PX459; Addgene) made by replacing the puromycin-resistant to a blasticidin-resistant gene. For construction of the targeting vector against murine SHMT2, the sgRNA sequences were determined using a web bioinformatics tool (https://benchling.com/academic). The oligo DNAs that encode the sgRNA were annealed and cloned into an AAV targeting vector (PX602; Addgene). The sgRNA sequences used are listed in Table S1.

For establishment of the 293A cell line with targeted deletion of SHMT, the targeting vector was transfected into cells using lipofectamine 2000 (Invitrogen). The drug-resistant cell clones were obtained by selecting the transfectants with $5 \mu \mathrm{g} / \mathrm{ml}$ of blasticidin and then were screened for loss of SHMT protein expression by Western blotting. For establishment of the AML12 cell line with targeted deletion of SHMT2, the adeno-assocaited virus serotype DJ/8 viral particles that harbor the CRISPR-Cas9 system were produced using the AAV Helper Free Packaging System (Cell Biolabs) and were then applied to the cells. The infectants were seeded into 96-well plates at the single-cell level using a flow cytometer. The cell clones were expanded and then screened for loss of SHMT2 protein expression by Western blotting. The genomic modifications were confirmed by automatic DNA sequencing.

For rescue of SHMT2 expression in the 293A mutant cell line, the full-length human SHMT2 cDNA was cloned into pcDNA3.1.puro, a modified vector of pcDNA3.1 (Invitrogen) made by replacing the G418-resistant with a puromycin-resistant gene. The synonymous mutations at the sgRNA binding site were introduced into the expression vector using a site-directed mutagenesis kit (Agilent) to evade gene targeting. The resultant SHMT2 expression vector was transfected into the SHMT2-knockout cells, and the drug-resistant clones were obtained by selection with $2 \mu \mathrm{g} / \mathrm{ml}$ of puromycin and were then screened for gain of SHMT2 protein expression by Western blotting.

\section{Western blotting}

Cells were washed twice in PBS and lysed in cytoplasmic lysis buffer (25 mM Tris- $\mathrm{HCl}$, pH 7.5, $40 \mathrm{mM} \mathrm{NaCl}$, and 1\% Triton X-100). Protein concentrations were determined with the Bradford reagent (BioRad). Cell lysates $(40 \mu \mathrm{g})$ were resolved by SDS-PAGE, and proteins were transferred onto nitrocellulose filters. The blots were saturated with 5\% nonfat milk and probed with antibodies against SHMT1 (\#HPA023314; Sigma-Aldrich, 1:1000), SHMT2 (\#HPA020543; SigmaAldrich, 1:1000), HK1 (\#2024; Cell Signaling Technology, 1:1000), lactate dehydrogenase A (\#3582; Cell Signaling Technology, 1:1000), PFKP (\#8164; Cell Signaling Technology, 1:1000), PKM1/2 (\#3190; Cell Signaling Technology, 1:1000), NDUFS1 (sc-271510; Santa Cruz, 1:1000), NDUFS3 (sc374282; Santa Cruz, 1:1000), $\beta$-actin (A2066; Sigma-Aldrich, 1:1000), or Total OXPHOS Human Antibody Cocktail (ab110411; Abcam, 1:1000). Following a wash with phosphate buffered saline with $0.1 \%$ Tween 20 , the blots were incubated with peroxidase-coupled goat anti-rabbit immunoglobulin G (Sigma-Aldrich, 1:5000). The immunolabeled protein bands were detected by enhanced chemiluminescence (ECL) method (Perkin Elmer). Densitometric analysis of the blots was performed using Image Quant TL software (GE Healthcare).

\section{Determination of cell proliferation and cell survival}

Cells were seeded on $6-\mathrm{cm}$ plates and grown for the indicated time intervals in the glucose or galactose media. The dead cells were excluded with trypan blue staining (Invitrogen) and the number of live cells was measured using a hemocytometer under a light microscope.

\section{Measurements of lactic acid, ATP, and basal oxygen consumption}

Measurement of lactic acid was adapted from Brandt et al (1980). In brief, appropriate amount of culture media was incubated at RT for $30 \mathrm{~min}$ in a final $100 \mu \mathrm{l}$ of reaction mix containing $160 \mathrm{mM}$ Trishydrazine, $\mathrm{pH}$ 9.0, $2.5 \mathrm{mM} \mathrm{NAD}+0.01 \% \mathrm{BSA}$, and $8 \mathrm{U}$ lactate dehydrogenase. The amount of lactic acid was extrapolated from a standard curve based on the Ab340 reading recorded on a microplate reader (BMG LABTECH). Cellular ATP level was determined using ENLITEN ATP assay kit (Promega) according to the manufacturer's specification. Intact cellular oxygen consumption was measured in the WT and $\triangle S H M T 2$ cells on an XFe24 seahorse bioanalyzer (Agilent) on plating $5 \times 10^{4}$ cells per well. Data have been represented as oxygen consumption relative to the WT cells.

\section{Determination of mRNA expression by next-generation sequencing and qRT-PCR}

Total cellular RNA was isolated using the Trizol reagent (Invitrogen). To determine the mRNA expression of the nuclear-encoded OXPHOS genes, CDNA libraries compatible for Illumina sequencing were prepared by using the QuantSeq 3' mRNA-seq Reverse (REV) Library Prep Kit (Lexogen) according to the manufacturer's instruction. The resultant cDNA libraries were assessed using a TapeStation (Agilent) and subjected to 100-bp single-end sequencing using the Illumina HiSeq 2500 system at the Wayne State University Applied Genomics Technology Center. Raw sequencing reads in FASTQ format were processed with Trimmomatic (Bolger et al, 2014) to remove low-quality and unknown sequences. To quantify transcript abundance, the processed reads in FASTA format were mapped to the hg19 human reference genome using bowtie2 (Langmead \& Salzberg, 2012). Transcript abundance in count per million was determined using express (Roberts \& Pachter, 2013), and differential gene expression was determined using edgeR (Robinson et al, 2010). 
To determine the mRNA expression of the mitochondriaencoded OXPHOS genes, CDNA libraries were constructed by random priming using the SuperScript III First-Strand Synthesis System (Invitrogen). The synthesized cDNA was used as a template for qRTPCR with SYBR Green qPCR Master Mixes (Thermo Scientific) on an M×3000P cycler (Stratagene). All mRNA levels were determined as the delta-delta threshold cycle $\left(\Delta \Delta C_{T}\right)$ and normalized to peptidylprolyl isomerase A mRNA level. The PCR primers used are listed in Table S2.

\section{Determination of mtDNA copy number}

mtDNA copy number was determined by calculating the ratio of the mitochondria-encoded ND1 gene levels to the nuclear-encoded $28 \mathrm{~S}$ rRNA gene levels using qPCR analysis. To quantify each gene level, serial dilutions of the cloned gene-specific PCR fragments were used to create a standard curve. The exact gene copy numbers of the ND2 and 28S rRNA genes were obtained by plotting the logtransformed $\triangle \triangle C_{T}$ values against the standard curves. The PCR primers used are listed in Table S2.

\section{Measurement of biosynthesis of the mitochondria-encoded proteins}

Cells growing on 6-cm plates to $90 \%$ confluency were metabolically labeled with $400 \mu \mathrm{Ci}$ EastTag EXPRESS35S Protein Labeling Mix (PerkinElmer) in the presence of $100 \mu \mathrm{g} / \mathrm{ml}$ emetine for $1 \mathrm{~h}$. Cell pellets were suspended in $1 \times$ Laemmli sample buffer and lysed by boiling for $10 \mathrm{~min}$. Equal amounts of the extracts were resolved on a $16.5 \%$ tricine gel and dried on a GelAir gel dryer (Bio-Rad). The radiolabeled proteins were visualized by a phosphorimager (Molecular Dynamics).

\section{Mitochondria isolation and BNGE}

To isolate mitochondria, cell suspensions were incubated in icecold homogenization buffer (10 mM Tris- $\mathrm{HCl}, \mathrm{pH} 7.5,250 \mathrm{mM}$ sucrose, and $1 \mathrm{mM}$ EDTA) for $15 \mathrm{~min}$ and then homogenized with a glass douncer by 15 strokes. After centrifugation at $600 \mathrm{~g}, 4^{\circ} \mathrm{C}$ for $10 \mathrm{~min}$, the supernatants were further centrifuged at $11,000 \mathrm{~g}, 4^{\circ} \mathrm{C}$ for $10 \mathrm{~min}$, to precipitate the mitochondrial fraction. Mitochondrial pellets were stored at $-80^{\circ} \mathrm{C}$ until use.

The BNGE was carried out as described by Schägger and von Jagow (1991) with minor modifications. Briefly, mitochondrial lysates were prepared by incubating mitochondrial suspension in the icecold lysis buffer (50 mM Tris- $\mathrm{HCl}, \mathrm{pH}$ 7.0, $750 \mathrm{mM}$ aminocapoic acid, and $1.7 \% \mathrm{n}$-dodecyl- $\beta$-D-moltoside) for $10 \mathrm{~min}$. The lysates were then clarified by centrifugation at $10,000 \mathrm{~g}, 4^{\circ} \mathrm{C}$ for $30 \mathrm{~min}$. The protein concentrations were determined using the bicinchoninic acid assay reagents (Pierce). The gel loading mixtures were prepared by adding 10x loading buffer (750 mM aminocapoic acid and $3 \%$ Coomassie blue brilliant G-250) to the $60 \mu \mathrm{g}$ of mitochondrial lysates. The BNGE was carried out by running the samples at $80 \mathrm{~V} /$ $150 \mathrm{~V}$ on a $3-12$ or $3 \%-16 \%$ gradient gel prepared from $41.6 \%, 100: 1$ acrylamide/ bis-acrylamide stock solution and $3 \times$ gel buffer $(150 \mathrm{mM}$ Bis-Tris, pH 7.0, and 1.5 M aminocaproic acid) and using separate anode (50 mM Bis-Tris, pH 7.0) and cathode (15 mM Bis-Tris and
$50 \mathrm{mM}$ tricine with or without $0.02 \%$ Coomassie blue brilliant $\mathrm{G}-250$ ) buffers. For Coomassie blue staining, the gels were fixed in a $50 \%$ methanol $/ 10 \%$ acetic acid solution, stained with $0.1 \%$ Coomassie blue R-250 in the fixing solution, and destained with a $40 \%$ methanol/ $10 \%$ acetic acid solution. For Western blotting, the resolved protein complexes were transferred onto polyvinylidene fluoride membrane and then probed with either NDUFS3 antibody or the Total OXPHOS antibody cocktail. For the in-gel activity assay, Complex I activity was developed by incubating the gel in a solution containing $50 \mathrm{mM}$ potassium phosphate buffer, $\mathrm{pH} 7.0,0.1 \mathrm{mg} / \mathrm{ml} \mathrm{NADH}$, and $0.2 \mathrm{mg} / \mathrm{ml}$ nitrotetrazolium blue.

\section{Supplemental Information}

Supplementary Information is available at https://doi.org/10.26508/ Isa.201800036.

\section{Acknowledgements}

We sincerely thank Drs. Todd Leff, James Granneman, and Shijie Sheng for critical comments on the manuscript.

\section{Author Contributions}

S Lucas: investigation.

$\mathrm{G}$ Chen: investigation.

S Aras: investigation and methodology.

J Wang: conceptualization, formal analysis, funding acquisition, investigation, methodology, and writing-original draft, review, and editing.

\section{Conflict of Interest Statement}

The authors declare that they have no conflict of interest.

\section{References}

Alberts B, Johnson A, Lewis J, Raff M, Roberts K, Walter P (2002) Molecular Biology of the Cell, pp 767-829. New York, NY: Garland Science.

Anderson DD, Quintero CM, Stover PJ (2011) Identification of a de novo thymidylate biosynthesis pathway in mammalian mitochondria. Proc Natl Acad Sci USA 108: 15163-15168. doi:10.1073/pnas.1103623108

Bao XR, Ong SE, Goldberger O, Peng J, Sharma R, Thompson DA, Vafai SB, Cox AG, Marutani E, Ichinose F (2016) Mitochondrial dysfunction remodels one-carbon metabolism in human cells. Elife 5: e10575. doi:10.7554/ elife.10575

Bolger AM, Lohse M, Usadel B (2014) Trimmomatic: A flexible trimmer for Illumina sequence data. Bioinformatics 30: 2114-2120. doi:10.1093/ bioinformatics / btu170

Bourdon A, Minai L, Serre V, Jais JP, Sarzi E, Aubert S, Chrétien D, de Lonlay P, Paquis-Flucklinger V, Arakawa H (2007) Mutation of RRM2B, encoding p53-controlled ribonucleotide reductase (p53R2), causes severe mitochondrial DNA depletion. Nat Genet 39: 776-780. doi:10.1038/ng2040 
Brandt RB, Siegel SA, Waters MG, Bloch MH (1980) Spectrophotometric assay for D-(-)-lactate in plasma. Anal Biochem 102: 39-46. doi:10.1016/ 0003-2697(80)90314-0

Brosnan ME, Brosnan JT (2016) Formate: The neglected member of onecarbon metabolism. Annu Rev Nutr 36: 369-388. doi:10.1146/annurevnutr-071715-050738

Chaneton B, Hillmann P, Zheng L, Martin AC, Maddocks OD, Chokkathukalam A, Coyle JE, Jankevics A, Holding FP, Vousden KH (2012) Serine is a natural ligand and allosteric activator of pyruvate kinase M2. Nature 491: 458-462. doi:10.1038/nature11540

de Koning TJ, Snell K, Duran M, Berger R, Surtees R (2003) L-serine in disease and development. Biochem J 371: 653-661. doi:10.1042/bj20021785

Ducker GS, Chen L, Morscher RJ, Ghergurovich JM, Esposito M, Teng X, Kang Y, Rabinowitz JD (2016) Reversal of cytosolic one-carbon flux compensates for loss of the mitochondrial folate pathway. Cell Metab 23: 1140-1153. doi:10.1016/j.cmet.2016.04.016

Ducker GS, Ghergurovich JM, Mainolfi N, Suri V, Jeong SK, Hsin-Jung Li S, Friedman A, Manfredi MG, Gitai Z, Kim H (2017) Human SHMT inhibitors reveal defective glycine import as a targetable metabolic vulnerability of diffuse large B-cell lymphoma. Proc Natl Acad Sci USA 114: 11404-11409. doi:10.1073/pnas.1706617114

Ducker GS, Rabinowitz JD (2017) One-carbon metabolism in health and disease. Cell Metab 25: 27-42. doi:10.1016/j.cmet.2016.08.009

Fiedorczuk K, Letts JA, Degliesposti G, Kaszuba K, Skehel M, Sazanov LA (2016) Atomic structure of the entire mammalian mitochondrial complex I. Nature 538: 406-410. doi:10.1038/nature19794

Guerrero-Castillo S, Baertling F, Kownatzki D, Wessels HJ, Arnold S, Brandt U, Nijtmans L (2017) The assembly pathway of mitochondrial Respiratory chain complex I. Cell Metab 25: 128-139. doi:10.1016/j.cmet.2016.09.002

Ingolia NT (2016) Ribosome footprint profiling of translation throughout the genome. Cell 165: 22-33. doi:10.1016/j.cell.2016.02.066

Kalhan SC, Hanson RW (2012) Resurgence of serine: An often neglected but indispensable amino acid. J Biol Chem 287: 19786-19791. doi:10.1074/ jbc.r112.357194

Kottakis F, Nicolay BN, Roumane A, Karnik R, Gu H, Nagle JM, Boukhali M, Hayward MC, Li YY, Chen T, et al (2016) LKB1 loss links serine metabolism to DNA methylation and tumorigenesis. Nature 539: 390-395. doi:10.1038/nature20132

Kozak M (1983) Comparison of initiation of protein synthesis in procaryotes, eucaryotes, and organelles. Microbiol Rev 47: 1-45.

Langmead B, Salzberg SL (2012) Fast gapped-read alignment with Bowtie 2. Nat Methods 9: 357-359. doi:10.1038/nmeth.1923

Locasale JW (2013) Serine, glycine and one-carbon units: Cancer metabolism in full circle. Nat Rev Cancer 13: 572-583. doi:10.1038/nrc3557

Mentch SJ, Mehrmohamadi M, Huang L, Liu X, Gupta D, Mattocks D, Gómez Padilla P, Ables G, Bamman MM, Thalacker-Mercer AE, et al (2015) Histone methylation dynamics and gene regulation occur through the sensing of one-carbon metabolism. Cell Metab 22: 861-873. doi:10.1016/j.cmet.2015.08.024

Minton DR, Nam M, McLaughlin DJ, Shin J, Bayraktar EC, Alvarez SW, Sviderskiy VO, Papagiannakopoulos T, Sabatini DM, Birsoy K (2018) Serine catabolism by SHMT2 is required for proper mitochondrial translation initiation and maintenance of formylmethionyl-tRNAs. Mol Cell 69: 610-621. doi:10.1016/j.molcel.2018.01.024

Morscher RJ, Ducker GS, Li SH, Mayer JA, Gitai Z, Sperl W, Rabinowitz JD (2018) Mitochondrial translation requires folate-dependent tRNA methylation. Nature 554: 128. doi:10.1038/nature25460

Neupert W (2016) Mitochondrial gene expression: A playground of evolutionary tinkering. Annu Rev Biochem 85: 65-76. doi:10.1146/ annurev-biochem-011116-110824
Nikiforov MA, Chandriani S, O'Connell B, Petrenko O, Kotenko I, Beavis A, Sedivy JM, Cole MD (2002) A functional screen for Myc-responsive genes reveals serine hydroxymethyltransferase: A major source of the one-carbon unit for cell metabolism. Mol cell Biol 22: 5793-5800. doi:10.1128/mcb.22.16.5793-5800.2002

Nikkanen J, Forsström S, Euro L, Paetau I, Kohnz Rebecca A, Wang L, Chilov D, Viinamäki J, Roivainen A, Marjamäki P, et al (2016) Mitochondrial DNA replication defects disturb cellular dNTP pools and remodel onecarbon metabolism. Cell Metab 23: 635-648. doi:10.1016/j. cmet.2016.01.019

Ott M, Amunts A, Brown A (2016) Organization and regulation of mitochondrial protein synthesis. Annu Rev Biochem 85: 77-101. doi:10.1146/annurev-biochem-060815-014334

Pfendner W, Pizer LI (1980) The metabolism of serine and glycine in mutant lines of Chinese hamster ovary cells. Arch Biochem Biophys 200: 503-512. doi:10.1016/0003-9861(80)90382-3

Ran FA, Hsu PD, Wright J, Agarwala V, Scott DA, Zhang F (2013) Genome engineering using the CRISPR-Cas9 system. Nat Protoc 8: 2281-2308. doi:10.1038/nprot.2013.143

Rhein VF, Carroll J, Ding S, Fearnley IM, Walker JE (2013) NDUFAF7 methylates arginine 85 in the NDUFS2 subunit of human complex I. J Biol Chem 288: 33016-33026. doi:10.1074/jbc.m113.518803

Roberts A, Pachter L (2013) Streaming fragment assignment for real-time analysis of sequencing experiments. Nat Methods 10: 71-73. doi:10.1038/nmeth.2251

Robinson BH, Petrova-Benedict R, Buncic JR, Wallace DC (1992) Nonviability of cells with oxidative defects in galactose medium: A screening test for affected patient fibroblasts. Biochem Med Metab Biol 48: 122-126. doi:10.1016/0885-4505(92)90056-5

Robinson MD, McCarthy DJ, Smyth GK (2010) edgeR: a Bioconductor package for differential expression analysis of digital gene expression data. Bioinformatics 26: 139-140. doi:10.1093/bioinformatics/btp616

Schägger H, von Jagow G (1991) Blue native electrophoresis for isolation of membrane protein complexes in enzymatically active form. Anal Biochem 199: 223-231. doi:10.1016/0003-2697(91)90094-a

Snell K, Natsumeda Y, Eble J, Glover J, Weber G (1988) Enzymic imbalance in serine metabolism in human colon carcinoma and rat sarcoma. $\mathrm{Br}$ Cancer 57: 87. doi:10.1038/bjc.1988.15

Stover P, Schirch V (1990) Serine hydroxymethyltransferase catalyzes the hydrolysis of 5, 10-methenyltetrahydrofolate to 5-formyltetrahydrofolate. J Biol Chem 265: 14227-14233

Stover PJ, Chen LH, Suh JR, Stover DM, Keyomarsi K, Shane B (1997) Molecular cloning, characterization, and regulation of the human mitochondrial serine hydroxymethyltransferase gene. I Biol Chem 272: 1842-1848. doi:10.1074/jbc.272.3.1842

Stroud DA, Surgenor EE, Formosa LE, Reljic B, Frazier AE, Dibley MG, Osellame LD, Stait T, Beilharz TH, Thorburn DR (2016) Accessory subunits are integral for assembly and function of human mitochondrial complex I. Nature 538: 123-126. doi:10.1038/nature19754

Suomalainen A, Battersby BJ (2018) Mitochondrial diseases: The contribution of organelle stress responses to pathology. Nat Rev Mol Cell Biol 19: 77-92. doi:10.1038/nrm.2017.66

Tani H, Ohnishi S, Shitara H, Mito T, Yamaguchi M, Yonekawa H, Hashizume O, Ishikawa K, Nakada K, Hayashi J-I (2018) Mice deficient in the Shmt2 gene have mitochondrial respiration defects and are embryonic lethal. Sci Rep 8: 425. doi:10.1038/s41598-017-18828-3

Tedeschi PM, Markert EK, Gounder M, Lin H, Dvorzhinski D, Dolfi S, Chan LL, Qiu J, DiPaola R, Hirshfield K (2013) Contribution of serine, folate and glycine metabolism to the ATP, NADPH and purine requirements of cancer cells. Cell Death Dis 4: e877. doi:10.1038/ cddis.2013.393 
Tibbetts AS, Appling DR (2010) Compartmentalization of Mammalian folatemediated one-carbon metabolism. Annu Rev Nutr 30: 57-81. doi:10.1146/annurev.nutr.012809.104810

Vazquez A, Markert EK, Oltvai ZN (2011) Serine biosynthesis with one carbon catabolism and the glycine cleavage system represents a novel pathway for ATP generation. PLoS One 6: e25881. doi:10.1371/journal. pone.0025881

Wang J, Alexander P, Wu L, Hammer R, Cleaver O, McKnight SL (2009) Dependence of mouse embryonic stem cells on threonine catabolism. Science 325: 435-439. doi:10.1126/science.1173288

Wu M, Neilson A, Swift AL, Moran R, Tamagnine J, Parslow D, Armistead S, Lemire K, Orrell J, Teich J (2007) Multiparameter metabolic analysis reveals a close link between attenuated mitochondrial bioenergetic function and enhanced glycolysis dependency in human tumor cells. Am J Physiol Cell Physiol 292: C125-C136. doi:10.1152/ ajpcell.00247.2006

Ye J, Mancuso A, Tong X, Ward PS, Fan J, Rabinowitz JD, Thompson CB (2012) Pyruvate kinase $M 2$ promotes de novo serine synthesis to sustain mTORC1 activity and cell proliferation. Proc Natl Acad Sci USA 109: 6904-6909. doi:10.1073/pnas.1204176109

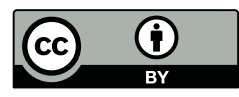

License: This article is available under a Creative Commons License (Attribution 4.0 International, as described at https://creativecommons.org/ licenses/by/4.0/). 